\title{
Statistical analysis plan for the EuroHYP-1 trial: European multicentre, randomised, phase III clinical trial of the therapeutic hypothermia plus best medical treatment versus best medical treatment alone for acute ischaemic stroke
}

Per Winkel ${ }^{1 *}$, Philip M. Bath², Christian Gluud', Jane Lindschou ${ }^{1 *}$ (D) H. Bart van der Worp ${ }^{3}$, Malcolm R. Macleod ${ }^{4}$, Istvan Szabo ${ }^{5}$, Isabelle Durand-Zaleski ${ }^{6}$, Stefan Schwab ${ }^{7}$ and for the EuroHYP-1 trial investigators

\begin{abstract}
Background: Cooling may reduce infarct size and improve neurological outcomes in patients with ischaemic stroke. In phase II trials, cooling awake patients with ischaemic stroke has been shown to be feasible and safe, but the effects in functional outcomes has not yet been investigated in an adequately sized randomised clinical trial.

Methods/design: The EuroHYP-1 trial is a multinational, randomised, superiority phase III clinical trial with masked outcome assessment testing the benefits and harms of therapeutic cooling in awake adult patients with acute ischaemic stroke. The outcomes dealt with here include the primary outcome the Rankin score (mRS) at day $91+/-14$ days after randomisation. The secondary and exploratory outcomes at day $91+/-14$ days unless otherwise stated encompassing: (1) death or dependency, defined as mRS score > 2; (2) death; (3) National Institutes of Health Stroke Score; (4) brain infarct size at $48+/-24$ hours; (5) EQ-5D-5 L score, and (6) WHODAS 2.0 score. Other outcomes are: the primary safety outcome serious adverse events; and the incremental cost-effectiveness, and cost utility ratios. The analysis sets include (1) the intention-to-treat population, and (2) the per protocol population. The sample size is estimated to 800 patients (5\% type 1 and 20\% type 2 errors). All analyses are adjusted for the protocol-specified stratification variables (nationality of centre), and the minimisation variables. In the analysis, we use ordinal regression (the primary outcome), logistic regression (binary outcomes), general linear model (continuous outcomes), and the Poisson or negative binomial model (rate outcomes).

Discussion: Major adjustments compared with the original statistical analysis plan encompass: (1) adjustment of analyses by nationality; (2) power calculations for the secondary outcomes; (3) to address the multiplicity problem using of a fixed-sequence testing procedure starting with the primary outcome followed by the secondary outcomes ordered according to falling power; (4) assignment of worst possible score to patients who are not alive at the planned date of measurement of the continuous scores; (5) improved imputations; (6) outline of a supplementary exploratory analysis of the temperature measurements and time to death; and (7) substantial reduction of sample size.
\end{abstract}

Trial registration: Clinicaltrials.gov, identifier: NCT01833312. 4 April 2013.

Keywords: Acute ischaemic stroke, Randomised clinical trial, Modified Ranking scale, Quality of life, Cooling, Cost-effectiveness

\footnotetext{
*Correspondence: per.winkel@ctu.dk; jane.lindschou@ctu.dk

${ }^{1}$ The Copenhagen Trial Unit, Centre for Clinical Intervention Research,

Blegdamsvej 9, Copenhagen, Denmark

Full list of author information is available at the end of the article
} 


\section{Introduction}

The EuroHYP-1 trial is a multicentre, randomised, superiority phase III international clinical trial with masked outcome assessment testing the benefits and harms of therapeutic cooling in awake adult patients with acute ischaemic stroke (website: http://www.eurohyp1.eu). The trial is designed according to the SPIRIT guidelines, and the background, design, and rationale have previously been published [1]. The EuroHYP-1 trial protocol has been available online on www.ClinicalTrials.gov since the start of the trial on 4 April 2013. The trial is endorsed and supported by European Clinical Research Infrastructures Network (ECRIN) (www.ecrin.org).

Here we describe the updated detailed statistical analysis plan that has been finalised while data collection in the EuroHYP-1 trial is underway, and to which all data analyses in the main publication of the EuroHYP-1 trial results will adhere. A detailed statistical analysis plan was part of the protocol, and the Steering Group of the EuroHYP-1 trial unanimously approved the statistical analysis plan on 30 March 2016. The present amendments of the original statistical analysis plan focus on the primary and the secondary outcomes. The amendments have been made in order to make the analysis more concrete and transparent. Patient recruitment of 800 patients is expected to be completed, and the final follow-up is predicted to occur in 2018-19, after which the database will be locked and data will be analysed.

\section{Methods}

\section{Objective of the EuroHYP-1 trial}

The primary aim of the EuroHYP-1 trial is to determine whether systemic cooling to a target temperature of $34-35{ }^{\circ} \mathrm{C}$, started within 6 hours of onset of stroke and maintained for up to 12 hours thereafter, improves outcomes in patients with acute ischaemic stroke. Inclusion and exclusion criteria are described in the design article in Tables 1 and 2 [1].

The null hypothesis is that there is no difference in death and disability as defined by a score on the modified Rankin Score (mRS) scale measured at $91+/$ - 14 days after randomisation of the patient.

For an overview of patient schedule and data collection see Fig. 1.

\section{The sample size}

Originally, we planned to demonstrate or reject an absolute difference of $7 \%$ between the intervention groups (equivalent to an odds ratio (OR) of 0.74 ) and allowing for 3\% loss to follow up in a sample size of 1500 randomised patients with a type 1 error risk of $5 \%$ and a type 2 error risk of $10 \%$ (EuroHYP-1, EudraCT number 2012-002944-25, version 2, 20 December 2012). Due to exceptionally slow enrolment, we realised that this target was no longer realistic. Accordingly, in 2014, the sample size was downgraded to 800 patients based on considerations explained in version 4 of the protocol (EuroHYP-1, EudraCT number 2012-002944-25, European Database on Medical Devices (EUDAMED) number CIV-12-09-008821, planned trial period July 2013 to March 2017 (45 months), version 4, date 29 June 2015). Currently, the project has been prolonged until 31 July 2018.

\section{Adjusting variables}

The randomisation is stratified according to the nationality of the participating centres and, within each

Table 1 Baseline characteristics of the participants in the EuroHYP-1 trial

\begin{tabular}{|c|c|c|c|c|}
\hline \multirow[t]{2}{*}{ Baseline characteristics } & \multicolumn{2}{|c|}{ Intention-to-treat population } & \multicolumn{2}{|c|}{ Per protocol population } \\
\hline & Intervention group & Control group & Intervention group & Control group \\
\hline \multicolumn{5}{|l|}{ Centre } \\
\hline \multicolumn{5}{|l|}{ - name of centre 1, N (\%) } \\
\hline \multicolumn{5}{|l|}{ - name of centre 2, N (\%) } \\
\hline \multicolumn{5}{|l|}{ - name of centre 3, N (\%) } \\
\hline \multicolumn{5}{|l|}{ - etc } \\
\hline \multicolumn{5}{|l|}{ Intention to perform thrombolysis, N (\%) } \\
\hline \multicolumn{5}{|l|}{ Surface cooling, N (\%) } \\
\hline \multicolumn{5}{|l|}{ Males, N (\%) } \\
\hline \multicolumn{5}{|l|}{ Stroke severity (NIHSS), mean (SD), N } \\
\hline \multicolumn{5}{|l|}{ Age $\leq 65$ years, $N(\%)$} \\
\hline \multicolumn{5}{|l|}{ Age, mean (SD), N } \\
\hline \multicolumn{5}{|l|}{ Visible ischaemic lesion on brain imaging, N (\%) } \\
\hline Time since symptom onset, $N \leq 4$ hours (\%) & & & & \\
\hline
\end{tabular}


Table 2 Power (based on an $a=0.05$ ) of the secondary outcomes and serious adverse events provided inclusion of 800 patients into the EuroHYP-1 trial

\begin{tabular}{|c|c|c|c|c|}
\hline Outcome & $\begin{array}{l}\text { Proportion or mean } \\
\text { value in control } \\
\text { group }\end{array}$ & $\begin{array}{l}\text { Standard deviation in the } \\
\text { control group for continuous } \\
\text { outcome }\end{array}$ & $\begin{array}{l}\text { Minimal relevant intervention } \\
\text { effect - absolute risk reduction } \\
\text { relative to control group }\end{array}$ & Power \\
\hline Score of NIHSS at $91+/-14$ days (sample size $=800$ ) & 8 points $^{a}$ & 5 points $^{a}$ & 2 points $^{a}$ & 1.00 \\
\hline $\begin{array}{l}\text { Serious adverse events at } 91+/-14 \text { days } \\
\text { (sample size }=800 \text { ) }\end{array}$ & $20 \%$ & NR & $10 \%$ & 0.98 \\
\hline $\begin{array}{l}\text { Death or dependency, defined as modified Rankin } \\
\text { score }>2 \text { at } 91+/-14 \text { days (sample size }=800 \text { ) }\end{array}$ & $63 \%$ & NR & $7.25 \%$ & 0.55 \\
\hline Brain infarct size at $48+/-24$ hours (sample size $=800$ ) & $10 \mathrm{ml}^{\mathrm{a}}$ & $15 \mathrm{ml}^{\mathrm{a}}$ & $2 \mathrm{ml}^{\mathrm{a}}$ & 0.47 \\
\hline EQ-5D-5 $L$ score at $91+/-14$ days (sample size $=800$ ) & 0.50 points & 0.40 points & 0.05 points & 0.42 \\
\hline Death at $91+/-14$ days (sample size $=800$ ) & $17 \%$ & NR & $3.84 \%$ & 0.33 \\
\hline
\end{tabular}

NIHSS National Institutes of Health Stroke Score, EQ-5D-5 L EuroQoL quality-of-life scale, NR not relevant

${ }^{a}$ Assumed values

stratum, the patient allocation is based on probabilistic minimisation (80:20) using the following factors:

- Intention to perform thrombolysis (yes compared to no).

- Method of cooling (surface compared to endovascular).

- Sex (male compared to female).

- Stroke severity (National Institutes of Health Stroke Score (NIHSS)) 6-12 compared to 13 or higher).

- Age ( $\leq 65$ years compared to $>65$ years).

- Visibility of a relevant ischaemic lesion on the first brain imaging (yes compared to no).

- Time since symptom onset ( $\leq 4$ hours compared to 4-6 hours).

The primary analyses will be adjusted by the protocolspecified stratification variable country and the seven minimisation factors.

\section{Flow of patients and baseline characteristics}

The flow of patients will be reported according to the CONSORT guidelines (Fig. 2). Patients' baseline characteristics will be reported in a table (Table 1) for the intentionto-treat population and for the per protocol population.

\section{Definition of the efficacy and safety outcomes}

Outcomes in the original statistical analysis plan (SAP) included in the EuroHYP-1 protocol are defined as primary, secondary, and exploratory. In the present SAP, we deal only with the primary and secondary outcomes as well as one safety outcome and one health-economic outcome. For information on the exploratory outcomes mentioned and defined in the EuroHYP-1 trial protocol and the analyses of these outcomes the reader is therefore referred to the protocol [1].

\section{The primary outcome}

The primary outcome is degree of disability post stroke as measured by the seven-level modified Rankin Scale measured in the period $91 \pm 14$ days after the patient's randomisation.

\section{The secondary outcomes originally selected}

The order of secondary outcomes as originally selected in the protocol was:

- Death or dependency, defined as a score on the $\mathrm{mRS}>2$ at outcome assessment in the period day $91 \pm 14$ days after randomisation. Type: binary.

- Death at outcome assessment in the period day $91 \pm$ 14 days after randomisation. Type: binary.

- Score on National Institute of Health Stroke Scale (NIHSS) at outcome assessment in the period day $91 \pm 14$ days after randomisation. Type: continuous.

- EuroQoL quality-of-life (EQ-5D-5 L) score at outcome assessment in the period day $91 \pm 14$ days after randomisation. Type: continuous.

- World Health Organization Disability Assessment Schedule II (WHODAS 2.0) score at outcome assessment in the period day $91 \pm 14$ days after randomisation. Type: continuous.

- Brain infarct size at imaging assessment in the period $48 \pm 24$ hours after randomisation. Type: continuous.

\section{Safety outcomes}

- Proportion of participants with at least one serious adverse event (SAE) until outcome assessment in the period day $91 \pm 14$ days after randomisation. Type: binary.

- Number of SAEs per patient until outcome assessment in the period day $91 \pm 14$ days after randomisation. Type: rate. 


\begin{tabular}{|c|c|c|c|c|c|c|c|c|c|c|c|}
\hline & A1 & \multicolumn{4}{|c|}{ TP } & A2 & A3 & A4 & A5 & A6 & A7/8 \\
\hline & Screening & & & & & $\begin{array}{c}\text { End of } \\
\text { hour } \\
24\end{array}$ & & Imaging & & & Outcome \\
\hline Day & & & & & & & & & & $\begin{array}{c}8 \text { or } \\
\text { discharge }\end{array}$ & $\begin{array}{c}91 \pm 14 \text { or } \\
\text { End of } \\
\text { trial }\end{array}$ \\
\hline Time & $\begin{array}{l}\text { Period of } \\
90 \mathrm{~min} \\
\text { prior to } \\
\text { start of } \\
\text { TP }\end{array}$ & $t=0$ & $\begin{array}{c}\text { Hour } \\
1-3: \\
\text { every } \\
15 \\
\min ^{18}\end{array}$ & $\begin{array}{l}\text { Hour } \\
4-12: \\
\text { every } \\
60 \text { min }\end{array}$ & $\begin{array}{l}\text { Hour } \\
4-12: \\
\text { every } \\
60 \\
\min ^{1}\end{array}$ & $\begin{array}{l}\text { Hour } \\
24 \pm 2\end{array}$ & $\begin{array}{l}\text { Hour } \\
48 \pm 2\end{array}$ & $\begin{array}{l}\text { Hour } \\
48 \pm 24\end{array}$ & $\begin{array}{l}\text { Hour } \\
72 \pm 4\end{array}$ & & \\
\hline $\begin{array}{l}\text { CT or MRI (standard of } \\
\text { care; may be performed } \\
\text { longer than } 90 \text { minutes } \\
\text { before start of treatment) }\end{array}$ & $x$ & & & & & & & $x$ & & & \\
\hline Patient enrolment ${ }^{16}$ & $\mathrm{X}$ & & & & & & & & & & \\
\hline Inclusion/exclusion criteria & $\mathrm{X}$ & & & & & & & & & & \\
\hline Demographics & $\mathrm{X}$ & & & & & & & & & & \\
\hline Medical history & $\mathrm{x}$ & & & & & & & & & & \\
\hline $\begin{array}{l}\text { Previous/concomitant } \\
\text { medication }^{2}\end{array}$ & $x$ & & & & & & & & & & \\
\hline Physical examination & $\mathrm{X}$ & & & & & $\mathrm{X}$ & $\mathrm{X}$ & & $\mathrm{X}$ & $\mathrm{X}$ & $\mathrm{X}$ \\
\hline $\begin{array}{l}\text { Body weight and height } \\
\text { (estimate) }\end{array}$ & $x$ & & & & & & & & & & \\
\hline 12-lead ECG & $\mathrm{X}$ & & & & & & & & & & \\
\hline Laboratory testing $^{3}$ & $\mathrm{X}$ & & & & & & & & & & \\
\hline $\begin{array}{l}\text { Pregnancy test (females of } \\
\text { childbearing potential only) }\end{array}$ & $x$ & & & & & & & & & & \\
\hline $\begin{array}{l}\text { Vital signs (blood pressure, } \\
\text { heart rate, respiratory rate) }\end{array}$ & $x$ & & $X^{19}$ & $\mathrm{x}$ & $\mathrm{X}^{1}$ & $\mathrm{x}$ & $\mathrm{x}$ & & $\mathrm{x}$ & $\mathrm{x}$ & $\mathrm{x}$ \\
\hline Oxygen saturation & $\mathrm{X}$ & & $\mathrm{X}^{19}$ & $\mathrm{X}$ & $\mathrm{X}^{1}$ & $\mathrm{X}$ & & & & & \\
\hline Body temperature ${ }^{4}$ & $\mathrm{X}$ & & $\mathrm{X}$ & $x$ & $\mathrm{X}^{1}$ & & & & K & & \\
\hline Modified Rankin Scale & $\mathrm{X}$ & & & & & & & & & $\mathrm{X}$ & $X^{5}$ \\
\hline NIHSS & $\mathrm{X}$ & & & & & $\mathrm{X}^{17}$ & $\mathrm{X}$ & & & $\mathrm{x}$ & $\mathrm{x}$ \\
\hline Randomisation & & $\mathrm{X}$ & & & & & & & & & \\
\hline Anti-shivering medication 6 & & $\mathrm{X}$ & & $\mathrm{X}$ & & & & & & & \\
\hline Induction of cooling ${ }^{7}$ & & $\mathrm{X}$ & & & & & & & & & \\
\hline Application of $\mathrm{IMDs}^{8}$ & & & & & & & & & & & \\
\hline Drug accountability & & & & & & & & & & & \\
\hline Inspection of insertion site ${ }^{9}$ & & & & & & & & & & & \\
\hline $\begin{array}{l}\text { Bedside Shivering } \\
\text { Assessment Scale }\end{array}$ & & & $\mathrm{x}$ & $\mathrm{x}$ & $x$ & & & & & & \\
\hline Patient survey & & & & & & & & & & $\mathrm{X}$ & $\mathrm{X}$ \\
\hline Monitoring for pneumonia ${ }^{10}$ & & & & & & K & & & & & \\
\hline $\begin{array}{l}\text { Assessment of antipyretic } \\
\text { medication }\end{array}$ & & & & & & k & & & & & \\
\hline $\begin{array}{l}\text { GCS motor response } \\
\text { subscale }\end{array}$ & $\mathrm{x}$ & & & & & & & & & & \\
\hline $\begin{array}{l}\text { Patient location during stay } \\
\text { in hospital } 11\end{array}$ & & & & & & & & & & & \\
\hline $\begin{array}{l}\text { Date/destination of } \\
\text { discharge }\end{array}$ & & & & & & & & & & $x$ & \\
\hline WHODAS 2.0 & & & & & & & & & & & $\overline{X^{12}}$ \\
\hline EQ-5D-5L & & & & & & & & & & & $\mathrm{X}^{12}$ \\
\hline $\begin{array}{l}\text { Health Recovery Guide } \\
\text { and Diary }\end{array}$ & & & & & & & & & & & $\mathrm{X}^{13}$ \\
\hline $\mathrm{AE} / \mathrm{SAE}$ assessment & & & & & & $\mathrm{X}$ & & & & & \\
\hline $\begin{array}{l}\text { Blood sampling for } \\
\text { biomarker }^{14}\end{array}$ & $\mathrm{x}$ & & & & & $x$ & & & $x$ & & \\
\hline Transcranial ultrasound ${ }^{15}$ & $\mathrm{X}$ & & & $\mathrm{X}$ & & & & & & & \\
\hline
\end{tabular}


(See figure on previous page.)

Fig. 1 Overview of patient schedule and data collection in the EuroHYP-1 trial (grey tone only for the experimental group). 1 Re-warming: hypothermia group only. 2 Previous medication including alteplase. 3 Includes sodium, potassium, magnesium, creatinine, urea, gamma-glutamyl transpeptidase, ASAT, ALAT, alkaline phosphatase, blood glucose; haemoglobin, haematocrit, erythrocytes, leukocytes, platelets, INR. Further samples may be taken throughout the study at the discretion of the investigator. 4 Body temperature will be assessed according to local clinical practice with tympanic, bladder, or rectal temperature measurement, except in patients randomised to therapeutic hypothermia from start of treatment phase (TP, beginning of hour 1) until end of re-warming period, when bladder or rectal thermal probes will be used. During TP, body temperature will be assessed every 15 min during the first 3 hours (except at time points $\mathrm{t}=0 \mathrm{~min}$ and $\mathrm{t}=15 \mathrm{~min}$ ) and every $60 \mathrm{~min}$ thereafter in patients randomised to therapeutic hypothermia, every 60 min (except at time point $\mathrm{t}=0 \mathrm{~min}$ ) in patients randomised to best medical treatment alone, subsequently in all patients at 8-hour intervals until A6 (day 8 or day of discharge from hospital, whichever occurs first). 5 The mRS assessment at outcome assessment (A7) will be recorded using a digital video camera. The clip will then be transferred to the EuroHYP-1 outcome adjudication web portal. 6 Anti-shivering medication: induction: buspirone 10 mg p.o./ pethidine $50 \mathrm{mg}$ i.v. $(2 \mathrm{~min}$ ); repeat doses of $10 \mathrm{mg}$ buspirone p.o. may be administered as long as a maximum dose of $30 \mathrm{mg} / 24 \mathrm{~h}$ is respected; a bolus of pethidine $25 \mathrm{mg}$ i.v. may be given as long as an interval of at least $30 \mathrm{~min}$ is respected and a maximum dose of $500 \mathrm{mg} / 24 \mathrm{~h}$ is not exceeded. $24 \mathrm{~h}$-doses include induction bolus. For the prevention and treatment of opioid-induced nausea and vomiting, a 5HT3RA may be administered as support medication. 7 Induction of cooling: $20 \mathrm{ml} / \mathrm{kg}$ estimated bodyweight $4{ }^{\circ} \mathrm{C}$ isotone saline or Ringer's lactate over a period of 30-60 min; EMCOOLS Brain.Pad, if available. $8 \mathrm{IMDs}$ permitted for cooling: EMCOOLS Brain.Pad (for induction of cooling only); Medivance/Bard Arctic Sun temperature management system with heat exchange control unit Arctic Sun 2000 or Arctic Sun 5000 and ArcticGel Pads; MTRE CritiCool temperature management system with heat exchange control unit CritiCool, accessoires and CureWrap; Zoll IVTM system with heat exchange control unit CoolGard 3000 or Thermogard XP, CoolGard start-up kit and intravascular temperature management catheters ICYy 3893 AE or ICY 3893 CO. 9 If endovascular cooling is performed, the catheter insertion site must be visually inspected for detection of bleeding/haematoma in 3-hour intervals during TP and once 3 hours after removal of the intravascular catheter. 10 Monitoring for pneumonia includes monitoring of oxygen saturation and body temperature, physical examination (auscultation, percussion) and, if clinically indicated, chest X-ray. Monitoring for signs of pneumonia must be performed from screening assessment (A1, within 90 minutes before the start of the treatment phase TP) until A6 (day 8 or day of discharge from hospital, whichever occurs first). 11 Patient location during stay in hospital must be assessed at 12:00 hours on each day in hospital. 12 WHODAS 2.0 questionnaire and EQ-5D questionnaire must be filled in by the patient or his/her relative/carer at outcome assessment (A7). 13 Health Recovery Guide and Diary: Section 6: filled in by the patient every day from discharge to V7; Section 7: filled in by the carer/relative prior to V7. 14 For participation in the biomarker sub-study a special informed consent form must be filled in by the patient or his/her legal representative. Assessment at End of Hour $24 \pm 2$ hours. 15 Only selected study sites. 16 Informed consent will be obtained in accordance with national regulatory requirements. 17 NIHSS assessment at End of Hour $24 \pm 2$ hours. 18 Starting at $\mathrm{t}=30 \mathrm{~min}$. 19 Every 60 minutes only. 20 Prior to intended repeat administration of pethidine. CT computed tomography, ECG electrocardiogram, EQ-5D-5 L EuroQoL quality-of-life scale, GCS Glasgow coma scale, MRI magnetic resonance imaging, NIHSS National Institutes of Health Stroke Score, SAE serious adverse event, WHODAS World Health Organization Disability Assessment Schedule

\section{Health economic outcome}

- Incremental cost utility (cost per quality-adjusted life years (QALY)) and incremental cost effectiveness (cost per death or disability averted) ratios. Type: continuous.

\section{The analysis sets}

The analysis populations for the statistical analyses of this trial include the intention-to-treat population, the per protocol set of patients, and the 'learning curve' population.

\section{The intention-to-treat population of patients}

The intention-to-treat (ITT) population is defined as all randomised patients classified according to the intervention to which they were randomised (experimental and control intervention groups) [2].

\section{The per protocol set of patients}

The per protocol set of patients is the subset of all randomised patients without major protocol violations and classified according to the intervention to which they were randomised. Major protocol deviations encompass: (1) patients in the intervention group who do not reach a body temperature of $\leq 35.0{ }^{\circ} \mathrm{C}$ within 3 hours after induction of cooling; or (2) patients randomised in the intervention group who do not achieve at least 6 hours of body temperature at a level of $\leq 35.0{ }^{\circ} \mathrm{C}$ during the period from beginning of hour 1 to the end of hour 12 of cooling. According to this definition, the per protocol population will be coded and defined in the data set before data lock, so it cannot undergo changes subsequently.

\section{The 'learning curve' population}

The EuroHYP-1 trial is a very large and complex trial with multiple nations and sites investigating a complex intervention. To account for this, we define a 'learning curve' analysis set, where we will analyse independently the first five participants included at each centre and compare those results to the results of all participants minus the first five.

\section{Statistical analyses Analytical principles}

Analyses will be conducted according to the intentionto-treat principle [2] if not otherwise stated.

All primary tests of significance will be two-sided with alpha $=5 \%$. Analyses will be conducted blinded with the two intervention groups coded as, e.g. 0 and 1. Two conclusions will be drawn, one assuming 0 is the intervention group and 1 is the control, and one assuming 


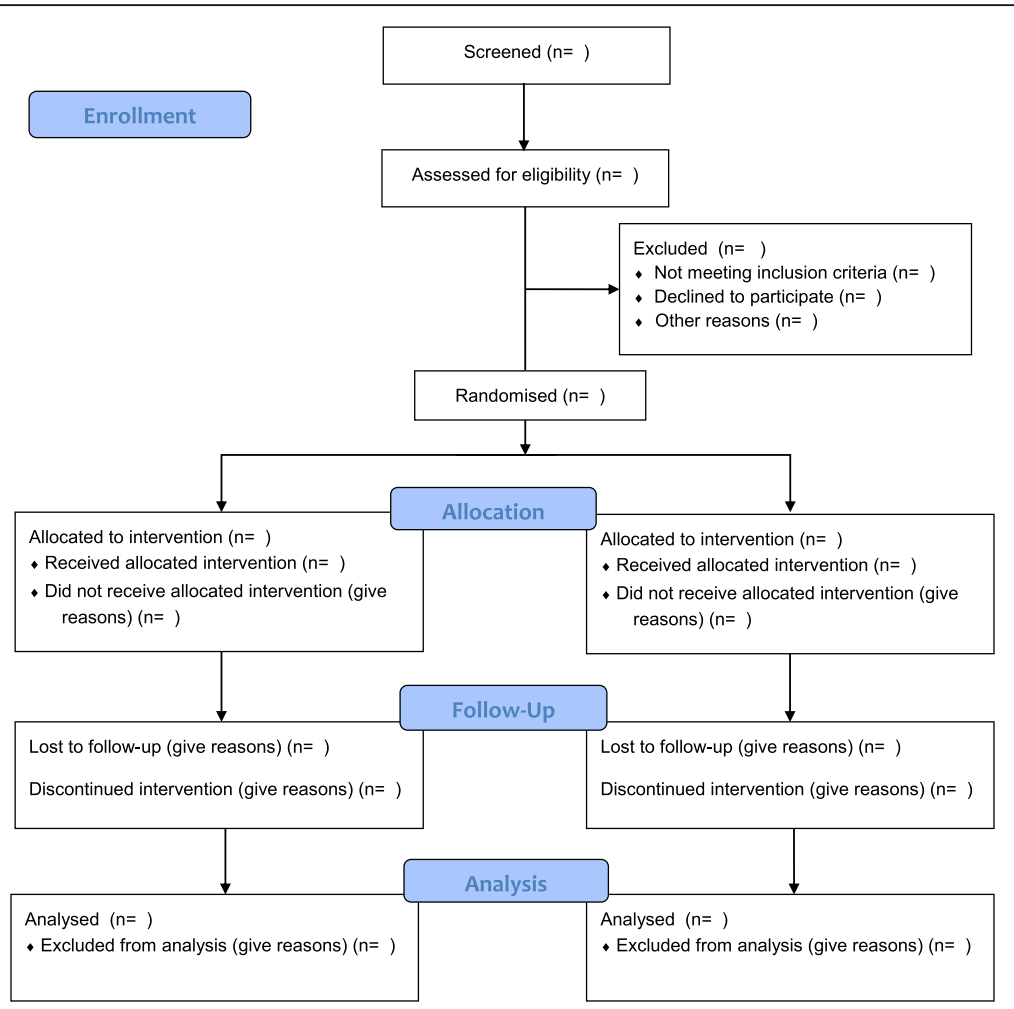

Fig. 2 Flow of participants in the EuroHYP-1 trial

the opposite. After two abstracts with two conclusions have been written and both accepted by the Steering Group, the code will be broken.

The primary analyses of primary, secondary, and exploratory outcomes will be those of the intention-totreat population adjusted for the protocol-specified stratification variable and the minimisation variables, and if necessary using data sets generated using multiple imputation.

\section{Missing values of the primary outcome}

By definition, none of the adjusting variables will have missing values (otherwise the stratification and minimisation could not have been conducted). So only the outcome value may be missing. Furthermore, we assume there are no useable auxiliary variable, i.e. no other variable that has a moderate to high correlation with the outcome $(|r|>0.40)$ and yet is not intended to be a predictor in the regression model. In this situation, the problem is easily solved. If we are willing to assume that the data are missing at random, the maximum likelihood analysis reduces to a complete case analysis $[3,4]$. Consequently, we simply delete participants that are missing on the outcome and estimate the regression with the remaining participants. However, if useable auxiliary variables have been identified, a multiple monotone imputation will be conducted, which will include the primary outcome and the covariates of the analytical model plus the auxiliary variables. As a sensitivity analysis to assess the potential impact of outcome values missing not at random (MNAR), we will conduct a worst/best case scenario imputation, i.e. a single value imputation of the missing values. The imputations use a minimum value or a maximum value (0 respectively 5 of the modified Rankin scale in that we assume that a patient who died will not have the mRS value missing). Missing values are imputed by the minimum value in one intervention group, and in the other intervention group by the maximum value and vice versa. Once the randomisation code is broken the results of the two comparisons may be interpreted.

\section{Power of the original secondary outcomes}

As the sample size is based on the primary outcome, we have calculated the power of each of the originally planned secondary outcomes given the sample size of 800 patients.

Death day $91 \pm 14$ days after randomisation: the implied clinically relevant minimal change in mortality that needs to be detected may be calculated based on (1) the mortality of $17 \%$ found in the meta-analysis quoted in the protocol [1]; (2) the assumption that wherever the mRS is dichotomised, the odds ratio for treatment versus control will be the same, the 'common odds ratio'; and (3) the requirement that this common odds ratio should not be larger 
than 0.74 (see the sample size calculation). Let the unknown change in mortality in the experimental group be $x$. If we assume the OR for the dichotomy $m R S>5$ compared to $\mathrm{mRS} \leq 5$ will be 0.74 and the mortality of the control group is $17 \%$ as quoted in the meta-analysis, we may find $\mathrm{x}$ by solving the equation $\mathrm{OR}=((0.17-\mathrm{x}) /(0.83+$ $\mathrm{x})) /(0.17 / 0.83)=0.74$ to obtain $\mathrm{x}=0.0384$ giving a mortality of $0.1700-0.0384=0.1316$ in the cooled patients versus 0.1700 in the controls. Using a sample size of 800 participants, the power is found to be 0.33 .

Death or dependency (mRS $>2) 91 \pm 14$ days after randomisation: using the same reasoning, the power of this outcome can be calculated by solving the equation $(0.63-\mathrm{x}) /(0.37+\mathrm{x})) / 0.63 / 0.37=(0.63-\mathrm{x}) 0.63 /(0.37+$ $\mathrm{x}) 0.37=0.74$ as $63 \%$ of the patients in the meta-analysis had $\mathrm{mRS}>2$ implying $\mathrm{x}=0.0725$. The power may then be calculated to be 0.55 .

NIHSS score $91 \pm 14$ days after randomisation: assuming a minimal relevant difference of 2 points, a standard deviation (SD) of 5 points [5], an $\alpha$ of 0.05 , and a sample size of 800 participants, the power may then be calculated to 1.00 .

EQ-5D-5 L score $91 \pm 14$ days after randomisation: assuming a minimal relevant difference of 0.05 points, a SD of 0.40 points [6-9], an $\alpha$ of 0.05 , and a sample size of 800 participants, the power may then be calculated to 0.42 .

WHODAS 2.0 score $91 \pm 14$ days after randomisation: we originally planned to analyse the World Health Organization Disability Assessment Schedule II (WHODAS 2.0) score at outcome assessment in the period day $91 \pm 14$ days after randomisation. However, due to difficulties in assessing the clinical meaning, we are not able to calculate the power, and we will thus analyse this as an exploratory outcome.

Brain infarct size $48 \pm 24$ hours after randomisation: assuming a minimal relevant difference of $2 \mathrm{ml}$, a SD of $15 \mathrm{ml}$ [10], an $\alpha$ of 0.05 , and a sample size of 250 participants, the power may then be calculated to 0.18 . Accordingly, this outcome will be exploratory.

Number of patients with at least one serious adverse events $91 \pm 14$ days after randomisation: assuming a control group event proportion of $20 \%$ of patients with at least one SAE, an intervention group event proportion of $10 \%$ [11], an $\alpha$ of 0.05 , and a sample size of 800 participants, the power may then be calculated to 0.98 .

Mean number of SAEs per patients: As we do not have prior data to indicate a probable minimal relevant difference, we cannot calculate power for this outcome. These data will be analysed as an exploratory outcome.

The results of calculations of the power are all shown in Table 2. These results are used to determine the sequence in which the secondary outcomes should be tested using the fixed-sequence approach, i.e. the sequence will be as follows:

1. Score on NIHSS at outcome assessment in the period day $91 \pm 14$ days after randomisation.

2. Proportion of participants with at least one serious adverse event (SAE) until outcome assessment in the period day $91 \pm 14$ days after randomisation.

3. Death or dependency, defined as a score on the $\mathrm{mRS}>2$ at outcome assessment in the period day $91 \pm 14$ days after randomisation.

4. Brain infarct size at imaging assessment in the period $48 \pm 24$ hours after randomisation.

5. EQ-5D-5 L score at outcome assessment in the period day $91 \pm 14$ days after randomisation.

6. Death at outcome assessment in the period day $91 \pm$ 14 days after randomisation.

The exploratory outcomes are:

1. WHODAS 2.0 score at outcome assessment in the period day $91 \pm 14$ days after randomisation.

2. Number of SAEs per patient until outcome assessment in the period day $91 \pm 14$ days after randomisation.

\section{Presentation of results in tables}

Tables 3, 4, 5 and 6 show how the results of the analysis of the primary outcome (Table 3 ), the binary secondary outcomes (Table 4), the continuous secondary outcome and exploratory outcomes (Table 5), and the SAE data (Table 6) will be presented.

Table 3 Comparison of the distributions of the modified Rankin scale (mRS) between the two intervention groups in each analysis set (intention-to-treat or per protocol)

\begin{tabular}{|c|c|c|c|c|c|c|c|c|c|c|}
\hline Population studied & $\begin{array}{l}\text { Intervention } \\
\text { group }\end{array}$ & $\begin{array}{l}\mathrm{mRS}=0 \\
\mathrm{~N}(\%)\end{array}$ & $\begin{array}{l}\mathrm{mRS}=1 \\
\mathrm{~N}(\%)\end{array}$ & $\begin{array}{l}\mathrm{mRS}=2 \\
\mathrm{~N}(\%)\end{array}$ & $\begin{array}{l}\mathrm{mRS}=3 \\
\mathrm{~N}(\%)\end{array}$ & $\begin{array}{l}\mathrm{mRS}=4 \\
\mathrm{~N}(\%)\end{array}$ & $\begin{array}{l}\mathrm{mRS}=5 \\
\mathrm{~N}(\%)\end{array}$ & $\begin{array}{l}\mathrm{mRS}=6 \\
\mathrm{~N}(\%)\end{array}$ & $\begin{array}{l}\text { Common OR } \\
(95 \% \mathrm{Cl})\end{array}$ & $\begin{array}{l}\text { P of difference } \\
\text { (reference = group 0) }\end{array}$ \\
\hline \multirow[t]{2}{*}{ Intention-to-treat (ITT) } & Group 0 & & & & & & & & & \\
\hline & Group 1 & & & & & & & & & \\
\hline \multirow[t]{2}{*}{ Per protocol (PP) } & Group 0 & & & & & & & & & \\
\hline & Group 1 & & & & & & & & & \\
\hline
\end{tabular}


Table 4 Comparison of the distributions of the secondary binary outcomes between the two intervention groups in each analysis set (intention-to-treat and per protocol)

\begin{tabular}{|c|c|c|c|c|c|c|c|}
\hline \multirow[t]{3}{*}{ Population } & \multirow{3}{*}{$\begin{array}{l}\text { Intervention } \\
\text { group }\end{array}$} & \multicolumn{6}{|c|}{ Outcomes } \\
\hline & & \multicolumn{3}{|l|}{ Death } & \multicolumn{3}{|c|}{$m R S>2$} \\
\hline & & $\mathrm{N}(\%)$ & Relative risk (RR) (95\% Cl) & P & $\mathrm{N}(\%)$ & RR $(95 \% \mathrm{Cl})$ & P \\
\hline \multirow[t]{2}{*}{ Intention-to-treat (ITT) } & Group 0 & & & & & & \\
\hline & Group 1 & & & & & & \\
\hline \multirow[t]{2}{*}{ Per protocol (PP) } & Group 0 & & & & & & \\
\hline & Group 1 & & & & & & \\
\hline
\end{tabular}

mRS modified Rankin score, $\mathrm{Cl}$ confidence interval

\section{Multiplicity}

We cannot claim a significant beneficial effect of the intervention in the trial if the primary outcome is neutral and a secondary outcome is statistically significant. Therefore, any testing addressing the multiplicity problem must not reduce the power of the test of the primary outcome as compared to the situation where we declare the secondary outcomes exploratory and only test the primary outcome using $\alpha=0.05$. Consequently, the primary outcome must be tested initially and using $\alpha=0.05$. These requirements may be fulfilled if we use a pre-specified testing sequence (fixed-sequence procedure) where each outcome is tested at $\alpha=0.05$ [12]. However, as soon as a non-significant result is obtained, the testing is stopped. However, for exploratory purposes we will calculate the $P$ values of the remaining tests and present them. We will use the fixedsequence procedure starting with the primary outcome and followed by the secondary outcomes, and the exploratory outcomes ordered according to descending power (see Table 2).

\section{Subgroup analysis}

The duration of the cooling intervention was reduced from 24 hours to 12 hours after the recruitment of 50 participants. To investigate this protocol change, we will conduct a subgroup analysis for the primary and secondary outcomes. In the analysis, we will compare the participants who were included before the protocol change to participants included after the protocol change.

\section{Sensitivity analyses}

The analyses of the primary and secondary outcomes will be repeated using the per protocol population and the 'learning curve' population.

Table 5 Comparison of distributions of the secondary continuous outcomes between the two intervention groups in each analysis set (intention-to-treat or per protocol)

\begin{tabular}{|c|c|c|c|c|c|c|c|c|c|}
\hline \multirow[t]{2}{*}{ Outcome } & \multirow[t]{2}{*}{ Population } & \multirow{2}{*}{$\begin{array}{l}\text { Intervention } \\
\text { group }\end{array}$} & \multicolumn{3}{|c|}{ Percentiles } & \multirow{2}{*}{ N } & \multirow{2}{*}{$\begin{array}{l}\text { Mean } \\
\text { (SD) }\end{array}$} & \multirow{2}{*}{$\begin{array}{l}\text { (Minimum, } \\
\text { maximum) }\end{array}$} & \multirow{2}{*}{$\begin{array}{l}P \text { of } \\
\text { difference }\end{array}$} \\
\hline & & & $25 \%$ & $50 \%$ & $75 \%$ & & & & \\
\hline \multirow{4}{*}{$\begin{array}{l}\text { NIHSS score Range of possible scores at } 91+/-14 \text { days } \\
\text { (RS): } 0 \text { to } 42 \text { Worst }=42\end{array}$} & \multirow[t]{2}{*}{ Intention-to-treat (ITT) } & Group 0 & & & & & & & \\
\hline & & Group 1 & & & & & & & \\
\hline & \multirow[t]{2}{*}{ Per protocol (PP) } & Group 0 & & & & & & & \\
\hline & & Group 1 & & & & & & & \\
\hline \multirow[t]{4}{*}{ EQ-5D-5 $L$ score at $91+/-14$ days RS: 0 to 100 Worst $=0$} & \multirow[t]{2}{*}{$\mathrm{ITT}$} & Group 0 & & & & & & & \\
\hline & & Group 1 & & & & & & & \\
\hline & \multirow[t]{2}{*}{ PP } & Group 0 & & & & & & & \\
\hline & & Group 1 & & & & & & & \\
\hline \multirow[t]{4}{*}{ Brain infarct size at $48+/-24$ hours Worst $=1$} & \multirow[t]{2}{*}{ ITT } & Group 0 & & & & & & & \\
\hline & & Group 1 & & & & & & & \\
\hline & \multirow[t]{2}{*}{ PP } & Group 0 & & & & & & & \\
\hline & & Group 1 & & & & & & & \\
\hline \multirow[t]{4}{*}{ WHODAS 2.0 score at $91+/-14$ days } & \multirow[t]{2}{*}{$\mathrm{ITT}$} & Group 0 & & & & & & & \\
\hline & & Group 1 & & & & & & & \\
\hline & \multirow[t]{2}{*}{ PP } & Group 0 & & & & & & & \\
\hline & & Group 1 & & & & & & & \\
\hline
\end{tabular}


Table 6 Amendments made relative to the original statistical analysis plan published in the protocol

\begin{tabular}{|c|c|c|}
\hline $\begin{array}{l}\text { Topic described in original } \\
\text { statistical analysis plan }\end{array}$ & $\begin{array}{l}\text { Handling of topic in the present } \\
\text { amended statistical analysis plan }\end{array}$ & Reason for action \\
\hline No adjustment for nationality. & Adjustment for nationality & $\begin{array}{l}\text { To improve the power by preventing upward } \\
\text { bias of the standard error of the outcome. }\end{array}$ \\
\hline $\begin{array}{l}\text { Power calculation of secondary } \\
\text { outcomes is missing }\end{array}$ & $\begin{array}{l}\text { Calculation of power conditional on } \\
\text { sample size }\end{array}$ & $\begin{array}{l}\text { To be used when defining the test ordering of } \\
\text { the secondary outcomes }\end{array}$ \\
\hline $\begin{array}{l}\text { Difficulties involved in interpreting an } \\
\text { effect on a secondary outcome that } \\
\text { can only be measured in surviving } \\
\text { patients was not addressed }\end{array}$ & $\begin{array}{l}\text { The worst possible score is assigned } \\
\text { to the dead patients }\end{array}$ & $\begin{array}{l}\text { A surplus of patients in one group relative to the } \\
\text { other group may die before the outcome is } \\
\text { measured. In the other group the corresponding } \\
\text { surviving patients may (or may not) have very } \\
\text { poor outcomes }\end{array}$ \\
\hline No multiplicity adjustment & $\begin{array}{l}\text { The fixed-sequence testing procedure } \\
\text { will be applied with the primary outcome } \\
\text { to be tested first and followed by the } \\
\text { secondary outcomes ordered according } \\
\text { to falling power }\end{array}$ & To keep the family-wise error rate $\leq 0.05$ \\
\hline $\begin{array}{l}\text { A search for auxiliary variables and if found } \\
\text { followed by imputation of the primary } \\
\text { outcome was not considered }\end{array}$ & $\begin{array}{l}\text { Missing value handling procedure } \\
\text { revised accordingly }\end{array}$ & $\begin{array}{l}\text { To improve the efficiency of a multiple imputation } \\
\text { of missing values of the primary outcome }\end{array}$ \\
\hline $\begin{array}{l}\text { The analytic potential of the exploratory } \\
\text { temperature data was not expanded on }\end{array}$ & $\begin{array}{l}\text { An outline of an exploratory mixed model } \\
\text { analyses of the temperature data and Cox } \\
\text { analyses of time to death with censoring } \\
\text { at end of treatment and at } 91 \text { days +/- } \\
14 \text { days is now included }\end{array}$ & $\begin{array}{l}\text { To assess if the temperature has an impact on } \\
\text { short-term and long-term mortality and supple } \\
\text { ment the result of the analysis of the second } \\
\text { secondary outcome }\end{array}$ \\
\hline Sample size of 1500 participants & Sample size reduced to 800 participants & $\begin{array}{l}\text { Due to exceptionally slow enrolment, we realised } \\
\text { that this target was no longer realistic }\end{array}$ \\
\hline
\end{tabular}

\section{Analysis of the primary and the secondary outcome The primary outcome}

The primary efficacy variable, the score on the mRS at the outcome assessment (day $91 \pm 14$ days), will be determined with ordinal (with more than two categories) logistic regression. The assumption about proportional odds will be accepted if the difference between the frequency in group 1 and that of group 2 in the various categories are all positive and different from 0 or are all negative and different from 0 . If the assumption of the ordinal regression analysis model is not fulfilled, the groups will be compared using a non-parametric method (van Elteren and stratification by nationality of centre) and the result will be the primary result. If the assumption of the model is fulfilled, the result of the adjusted analysis will be the primary result.

\section{The secondary and the exploratory outcomes}

Frequencies and percentages per group as well as risk ratios with $95 \%$ confidence interval (CI) will be reported for binary outcomes. Continuous variables and rate variables will be summarised using mean, standard deviation, 25, 50 and 75 percentiles, and minimum and maximum values.

Logistic regression for binary quantities, the general linear univariate model for continuous outcomes, and the Poisson distribution or negative binomial distribution for rate outcomes will be used. If the assumptions of the Poisson or negative binomial models are not fulfilled with reasonable approximation, a non-parametric method (van Elteren adjusted by nationality of centre) will be used.

\section{Outline of exploratory analysis of the temperature variables and time to death}

Assuming that the temperature is measured at the same times relative to the time of the start of intervention, a mixed model may be used to characterise the time course of those patients who do not die during the treatment. The model is given by temperature =INT time time*INT, where INT is the intervention indicator. Additionally, the time to death from start of treatment with censoring at end of treatment and at 91 days $+/-14$ days including baseline variables measured at or prior to start of treatment may be analysed using a Cox proportional hazards model. The mean values of all the actual measured temperatures in the intervention group will be displayed in a graph with mean $+/-2$ standard errors.

\section{Health economic evaluation}

The economic assessments will be conducted in alignment with the Consolidated Health Economic Evaluation Reporting Standards (CHEERS) statement [13]. The prospective analysis will determine the cost per QALY gained with systemic cooling compared with standard care over a 3-month period. Acute hospital postdischarge resources will be considered in the evaluation. Procedural costs for systemic cooling will be obtained with a bottom-up micro-costing approach in order to 
identify all relevant cost components of the procedure and value each component for all individual patients, using procedure duration, staff, and number of medical devices as variables. All-cause hospital admissions within 3 months of the first treatment will also be included in cost computations. Non-hospital resources are not considered in view of the short follow-up duration.

Cost of systemic cooling will be the manufacturer's price, and staff costs will be estimated from gross salaries. Hospital inpatient costs will be estimated using current average national cost of each patient's diagnosisrelated group (DRG), adjusted for actual length of stay, and resources consumed during hospitalisation. Hospital re-admissions will be based on tariffs. All costs will be in Euros $(€)$ and are not discounted. Health-related quality of life collected using the EQ-5D-5 L self-administered questionnaire at baseline and 3 months will be used to elicit utility values based on country-specific tariffs. The difference in QALYs will be estimated as the difference in the area between the utility curves for the two groups.

We will undertake a fully pooled analysis for clinical outcomes and a country-specific analysis for costs (based on the countries with the highest patient volume) [14].

The cost-utility analysis will estimate incremental costs per QALY and the cost-effectiveness incremental cost per death or disability (as defined in the secondary outcomes) averted. Between-group comparisons of QALYs and costs will be performed with the statistical test appropriate for their distribution, with a significance threshold of 0.05 . A joint comparison of costs and effects will be performed by nonparametric bootstrapping with 1000 resamples and the result of the bootstrap replications presented on the cost-effectiveness plane (scatterplot).

\section{Overview of changes to the original statistical analysis plan for the primary and the secondary outcomes}

Table 7 presents an overview of the changes relative to the original statistical analysis plan and explain the reasoning behind. The only substantial changes relative to the original plan includes that we now: (1) adjust the analyses by the protocol-specified stratification variable, i.e. nationality to improve the power [15]; (2) power calculations for secondary outcomes; (3) address the multiplicity problem by using a fixed-sequence procedure starting with the primary outcome followed by the secondary outcomes ordered according to falling power; (4) due to the low power involved in detecting small (1-5\%) differences in mortality between the groups, assign worst possible score to patients who are not alive at the planned date of measurement of the continuous scores; (5) include auxiliary variables in the potential multiple imputation of missing value of the primary outcome to improve the efficiency of the imputation; and (6) outline a supplementary exploratory analysis of the temperature measurements and time to death; (7) substantial reduction of sample size.

\section{Discussion}

With this updated detailed statistical analysis plan we present the different analyses in the main publication of the EuroHYP-1 trial to avoid risks of outcome reporting bias and data driven results $[16,17]$. Of the pre-specified outcomes in the trial, we choose to report only the primary outcome, the secondary outcome, two exploratory outcomes including serious adverse events in the main publication because of the complexity of the remaining safety variables and the detailed economical quantities that require separate publications.

\section{The interpretation of the effect on outcomes which may only be measured in surviving patients}

Formally, three of the original secondary outcomes (NIHSS, EQ-5D-5 L, and infarct size) are only measurable in patients who survive until these outcomes were planned to be measured. This may make the interpretation of a significant effect on each of the last four secondary outcomes somewhat difficult when the power of detecting a small difference between the mortalities in the two groups is low. A surplus of patients in one intervention group relative to the other group may die before the outcome is

Table 7 Total number of serious adverse events (SAEs), and number of patients with at least one SAE groups in each analysis set (intention-to-treat or per protocol)

\begin{tabular}{|c|c|c|c|c|c|c|}
\hline \multirow{2}{*}{$\begin{array}{l}\text { Types of } \\
\text { events }\end{array}$} & \multicolumn{3}{|l|}{ Group 0} & \multicolumn{3}{|l|}{ Group 1} \\
\hline & Events, N & Patients with at least one event, $\mathrm{N}$ & Patients assessed, $\mathrm{N}$ & Events, N & Patients with at least one event, $\mathrm{N}$ & Patients assessed, N \\
\hline \multicolumn{7}{|l|}{ Any event } \\
\hline \multicolumn{7}{|l|}{$\begin{array}{l}\text { Event type } \\
1\end{array}$} \\
\hline \multicolumn{7}{|l|}{$\begin{array}{l}\text { Event type } \\
2\end{array}$} \\
\hline \multicolumn{7}{|l|}{$\begin{array}{l}\text { Event type } \\
3\end{array}$} \\
\hline Etc & & & & & & \\
\hline
\end{tabular}


measured. In the other intervention group, the corresponding surviving patients may (or may not) have very poor outcome values which may make the first group look good if the mortalities do not differ significantly.

Given the sample size of 800 patients and assuming the mortality in one group is 0.17 (e.g. in the control group, see above) the probability of not detecting an absolute reduction in mortality in the experimental group of 0.01 , $0.02,0.03,0.04$, and 0.05 is $0.93,0.89,0.81,0.70$, and 0.57 , respectively. Thus, differences in mortality of the intervention groups of $5 \%$ or less are likely to remain undetected with a relatively high probability. Therefore, we have elected to include the dead patients in the comparison between the two intervention groups by assigning the worst possible score to the dead patients. The EQ5D5L is used to estimate QALYs gained in each arm of the trial population. QALYs are estimated by summing up the time spent in each state multiplied by the quality of life attributed to this state. By definition, the value of QALYs is zero from the time of death onwards. Total QALYs will be computed and reported for each patient for the entire duration of the follow-up.

For the study of prognostic markers in the EuroHYP-1 trial, we will apply similar principles as in the Clarithromycin in Patients with Stable Coronary Heart Disease trial (CLARICOR) trial [18]. The study of prognostic markers will not be part of the primary publication.

In conclusion, major adjustments compared with the original statistical analysis plan encompass: (1) adjustment of analyses by nationality; (2) power calculations for the secondary outcomes; (3) use of a fixed-sequence testing procedure starting with the primary outcome followed by the secondary outcomes ordered according to falling power to address the multiplicity problem; (4) assignment of worst possible score to patients who are not alive at the planned date of measurement of the continuous scores; (5) improved imputations; (6) outline of a supplementary exploratory analysis of the temperature measurements and time to death; and (7) substantial reduction of sample size.

\section{Additional file}

Additional file 1: List of principal investigators in EuroHYP-1 and sites. (DOCX $32 \mathrm{~kb})$

\section{Acknowledgements}

We thank Gordon D. Murray for critical comments and helpful discussions on earlier versions of this article. Please find the complete list of the EuroHYP-1 investigators in Additional file 1.

\section{Funding}

EuroHYP-1 has received funding from the European Union's Seventh Framework Programme (FP7/2007-2013) under grant agreement no. 278709. The funding body has no role in the design of the trial and data collection, analysis, interpretation of data, and in writing the manuscript.

\section{Availability of data and materials}

Pseudo-anonymised data from the EuroHYP-1 will be made publicly available in Zenodo (https://zenodo.org/) after completion of data analyses.

\section{Authors' contributions}

PW and CG proposed the statistical analysis plan. PW drafted the manuscript. PW, PMB, CG, JL, HBW, MRM, IS, IDZ and SS participated in the design and coordination of the trial. PW, PMB, CG, JL, HBW, MRM, IS, IDZ and SS read, amended, and approved the statistical analysis plan and the final manuscript. All authors read and approved the final manuscript.

\section{Authors' information}

Not applicable.

\section{Ethics approval and consent to participate}

All included participants have provided verbal and written consent before inclusion in the trial. The trial has been approved by the relevant ethics committees in all participating countries (Belgium (AZ Sint Jan BruggeOostende AV Ethics Committee OG 065, ref no. 1759, 7 May 2015), Denmark (Regional Ethics Committee for Region Hovedstaden, ref no. 30113713, 10 Oct 2013), Finland (Hospital District of South-West Finland, ref no. 161/1800/ 2014, 28 Jun 2016), France (Comité de Protection des Personnes Nord Ouest IV, ref no. CPP 14/21, 13 Jun 2014), Germany (Ethik-Kommission der FAU, Erlangen, ref no 63_13 Az and 67_13 Mz, 7 Jun 2013), Ireland (SJH/AMNCH Research Ethics Committee, ref no. 2015-11 List 43 (4), 26 Nov 2915), Italy (Comitato Etico Dell' Universita' "Sapienza", ref no. 161 SA/2016, 8 May 2015), Lithuania (Lietuvos Bioetikos Komitetas, ref no. L-15-04/1, 18 Feb 2015), Poland (Instytut Psychiatrii I Neurologii Komisja Bioetyczna, ref no. 8/2014, 12 Jun 2014), Spain (CEIC del Hospital Universitari Vall d'Hebrón, 4 Apr 2014), Sweden (Regionala Etikprovningsnamden Lund, ref no. 2016/1088, 2 Feb 2017), Turkey (Hacettepe Universitesi Klinik Arastirmalar Etik Kurulu Karar Formu, ref no. KA 14039, 19 Mar 2015), UK England (North East - Newcastle \& North Tyneside 2 Research Ethics Committee, ref no. 3/NE/0299, 2 Dec 2013), UK Scotland (Scotland A Research Ethics Committee, ref no. 13/SS/0194, 9 Dec 2013).

\section{Consent for publication}

Not applicable (no individual details will be published based from the EuroHYP-1 trial, only summary data will be used, thus assuring the anonymity of the trial participants).

\section{Competing interests}

The authors declare that they have no competing interests.

\section{Publisher's Note}

Springer Nature remains neutral with regard to jurisdictional claims in published maps and institutional affiliations.

\footnotetext{
Author details

${ }^{1}$ The Copenhagen Trial Unit, Centre for Clinical Intervention Research, Blegdamsvej 9, Copenhagen, Denmark. ${ }^{2}$ Stroke Trials Unit, Division of Clinical Neuroscience, University of Nottingham, Nottingham NG5 1PB, UK. ${ }^{3}$ Department of Neurology and Neurosurgery, Brain Center Rudolf Magnus, University Medical Center Utrecht, Heidelberglaan 100, 3584 CX Utrecht, The Netherlands. ${ }^{4}$ Division of Clinical Neurosciences, University of Edinburgh, FU204, Chancellor's Building, Royal Infirmary of Edinburgh, Little France Crescent, Edinburgh EH16 4SA, UK. ${ }^{5}$ European Stroke Research Network for Hypothermia, Square de Meeus 38/40, 1000 Brussels, Belgium. ${ }^{6}$ ECEVE, UMR 1123, URCEcolle de France Hôpital de l'Hotel Dieu, 1 Place du parvis de Notre Dame, F 75004 Paris, France. ${ }^{7}$ Neurologische Klinik,
} 
Universitætsklinikum Erlangen, Schwabachanlage 6, 91054 Erlangen, Germany.

Received: 10 March 2017 Accepted: 25 October 2017

Published online: 29 November 2017

\section{References}

1. van der Worp HB, Macleod MR, Bath PM, Demotes J, Durand-Zaleski I, Gebhardt B, Gluud C, Kollmar R, Krieger DW, Lees KR, Molina C, Montaner J, Roine RO, Petersson J, Staykov D, Szabo I, Wardlaw JM, Schwab S. EuroHYP1: European multicenter, randomized, phase III clinical trial of therapeutic hypothermia plus best medical treatment vs. best medical treatment alone for acute ischemic stroke. Int J Stroke. 2014;9(5):642-5.

2. Fergusson D, Aaron SD, Guyatt G, Hebert P. Post-randomisation exclusions: the intention to treat principle and excluding patients from analysis. BMJ. 2002;325(7365):652-4.

3. Allison DB. Handling missing data by maximum likelihood. SAS Global Forum; 2012. http://www.statisticalhorizons.com/wp-content/uploads/ MissingDataByML.pdf. Accessed 13 Nov 2017.

4. Carpenter JR, Kenward MG. Multiple imputation and its application. Statistics in practice. Chichester: Wiley; 2013.

5. The National Institute of Neurological Disorders and Stroke rt-PA Stroke Study Group. Tissue plasminogen activator for acute ischemic stroke. N Engl J Med. 1995:333(24):1581-7.

6. CLOTS (Clots in Legs Or sTockings after Stroke) Trials Collaboration. Effect of intermittent pneumatic compression on disability, living circumstances, quality of life, and hospital costs after stroke: secondary analyses from CLOTS 3, a randomised trial. Lancet Neurol. 2014;13(12):1186-92.

7. Dennis M, Sandercock P, Reid J, Graham C, Murray G, Venables G, Rudd A, Bowler $\mathrm{G}$. The effect of graduated compression stockings on long-term outcomes after stroke: the CLOTS trials 1 and 2. Stroke. 2013:44(4):1075-9.

8. Rangaraju S, Frankel M, Jovin TG. Prognostic value of the 24-hour neurological examination in anterior circulation ischemic stroke: a post hoc analysis of two randomized controlled stroke trials. Interv Neurol. 2016:4(3-4):120-9.

9. The IST-3 collaborative group. Effect of thrombolysis with alteplase within 6 h of acute ischaemic stroke on long-term outcomes (the third International Stroke Trial [IST-3]): 18-month follow-up of a randomised controlled trial. Lancet Neurol. 2013;12(8):768-76.

10. Krongold M, Almekhlafi MA, Demchuk AM, Coutts SB, Frayne R, Eilaghi A. Final infarct volume estimation on 1-week follow-up MR imaging is feasible and is dependent on recanalization status. Neuroimage Clin. 2014;7:1-6.

11. Hemmen TM, Raman R, Guluma KZ, Meyer BC, Gomes JA, Cruz-Flores S, Wijman CA, Rapp KS, Grotta JC, Lyden PD. Intravenous thrombolysis plus hypothermia for acute treatment of ischemic stroke (ICTUS-L): final results. Stroke. 2010:41(10):2265-70.

12. Dmitrienko A, Tamhane AC, Bretz F. Multiple testing problems in pharmaceutical statistics. Boca Raton: CRC Biostatistics Series: Chapman and Hall; 2010. p. 52-70.

13. Husereau D, Drummond M, Petrou S, Carswell C, Moher D, Greenberg D, Augustovski F, Briggs AH, Mauskopf J, Loder E. Consolidated Health Economic Evaluation Reporting Standards (CHEERS)-explanation and elaboration: a report of the ISPOR Health Economic Evaluation Publication Guidelines Good Reporting Practices Task Force. Value Health. 2013;16(2):231-50.

14. Raikou M, Briggs A, Gray A, McGuire A. Centre-specific or average unit costs in multi-centre studies? Some theory and simulation. Health Econ. 2000;9(3):191-8

15. Kahan BC, Morris TP. Improper analysis of trials randomised using stratified blocks or minimisation. Stat Med. 2012;31(4):328-40.

16. Dwan K, Gamble C, Williamson PR, Altman DG. Reporting of clinical trials: a review of research funders' guidelines. Trials. 2008;9:66.

17. Finfer S, Bellomo R. Why publish statistical analysis plans? Crit Care Resusc. 2009;11(1):5-8

18. Winkel P, Jakobsen JC, Hilden J, Lange T, Jensen G, Kjøller E, Sajadiah A, Kastrup J, Kolmos HJ, Larsson A, Arnlov A, Gluud C. Predictors for major cardiovascular outcomes in stable ischaemic heart disease (PREMAC): Statistical analysis plan for data originating from the CLARICOR (clarithromycin for patients with stable coronary heart disease) trial. Diagn Prognost Res. 2017;1:1-10.

\section{Submit your next manuscript to BioMed Central and we will help you at every step:}

- We accept pre-submission inquiries

- Our selector tool helps you to find the most relevant journal

- We provide round the clock customer support

- Convenient online submission

- Thorough peer review

- Inclusion in PubMed and all major indexing services

- Maximum visibility for your research

Submit your manuscript at www.biomedcentral.com/submit 